\title{
Large zero point density fluctuations in fluids
}

\author{
Peter Wu $\oplus^{*}$ and L. H. Ford $\oplus^{\dagger}$ \\ Institute of Cosmology, Department of Physics and Astronomy, Tufts University, Medford, Massachusetts 02155, USA
}

(Received 8 May 2020; accepted 9 July 2020; published 29 July 2020)

\begin{abstract}
Zero point density fluctuations in a liquid and their potential observation by light scattering are discussed. It is suggested that there are two distinct effects of interest. One gives an average number of scattered photons, and depends upon an inverse power of the photon wavelength. The second effect arises in the scattering of finite size photon wave packets and depends upon an inverse power of the spatial size of the wave packet, as well as upon the shape of the wave packet. This effect appears as large fluctuations in the number of scattered photons, and is analogous to the vacuum fluctuations of space-time averages of the energy density in quantum field theory. It is an illustration that quantum fluctuations become larger when probed on smaller length and time scales.
\end{abstract}

DOI: 10.1103/PhysRevResearch.2.032028

Zero point fluctuations play an important role in several areas of physics, including both condensed matter physics and quantum field theory. These fluctuations are a direct consequence of the uncertainty principle. When a quantum system is observed on smaller length or time scales, the zero point fluctuations become greater. In quantum field theory, this means that the operators describing observable quantities must be averaged over finite regions. The electric field or energy density at a single space-time point is not meaningful, but a space and time average is. This fact has a deep connection with quantum measurement and the principle that only observable quantities are physically meaningful. Any measurement of a field operator necessarily involves an average over finite space and time regions.

The quantum fluctuations of a quadratic field operator, such as energy density, are especially subtle, with a probability distribution which falls more slowly than exponentially [1-3]. This leads to an enhanced probability for very large vacuum fluctuations for quantum stress tensors, which can in turn drive large fluctuations of the gravitational field, a variety of quantum gravity effect [4].

The zero point fluctuations in the density of a fluid provides an analog model for quantum stress tensor fluctuations, as well as being an interesting phenomenon in its own right. We can write the local mass density of the fluid as

$$
\hat{\rho}(t, \mathbf{x})=\rho_{0}+\hat{\rho}_{1}(t, \mathbf{x}),
$$

where $\rho_{0}$ is the average mass density, and $\hat{\rho}_{1}(t, \mathbf{x})$ is an operator which describes the local density fluctuations around the mean value. This operator may be expanded in terms of

\footnotetext{
*peter.wu610348@tufts.edu

†ford@cosmos.phy.tufts.edu
}

Published by the American Physical Society under the terms of the Creative Commons Attribution 4.0 International license. Further distribution of this work must maintain attribution to the author(s) and the published article's title, journal citation, and DOI. phonon creation and annihilation operators as [5]

$$
\hat{\rho}_{1}(t, \mathbf{x})=\sum_{\mathbf{q}} \sqrt{\frac{\rho_{0} \hbar \omega}{2 V c_{s}^{2}}}\left[e^{i(\mathbf{q} \cdot \mathbf{x}-\omega t)} b_{\mathbf{q}}+e^{-i(\mathbf{q} \cdot \mathbf{x}-\omega t)} b_{\mathbf{q}}^{\dagger}\right],
$$

where $V$ is a quantization volume and $\omega=c_{s}|\mathbf{q}|$, with $c_{s}$ the speed of sound in the fluid. This operator is proportional to the time derivative of a massless scalar field [6,7], $\phi$ :

$$
\hat{\rho}_{1}(t, \mathbf{x})=\frac{\sqrt{\rho_{0}}}{c_{s}^{2}} \dot{\phi}(t, \mathbf{x}) .
$$

Here $\phi$ is identical to the relativistic massless scalar field, except with the speed of light replaced by the speed of sound, $c \rightarrow c_{s}$. Thus the fluctuations of $\hat{\rho}_{1}$ may be understood from those of $\dot{\phi}$.

The density fluctuations are potentially observable in lightscattering experiments. This effect was discussed in Ref. [8], where the differential cross section for scattering by the zero point density fluctuations was derived. Integrating over scattering angle, summing over final photon polarizations, and averaging on initial polarizations convert this result into a total cross section

$$
\sigma_{0}=\frac{368 \pi^{4}}{105} \frac{\hbar \mathcal{V} \eta^{4}}{c_{s} \rho_{0} \lambda_{0}^{5}}
$$

Here $\lambda_{0}$ is the vacuum wavelength of the light, $\eta$ is the index of refraction for the fluid, and $\mathcal{V}$ is the effective scattering volume of the fluid. As discussed in Ref. [8], this effect may be viewed as Brillouin scattering by the density fluctuations, and the $\lambda_{0}^{-5}$ dependence may be viewed as a product of a factor of $\lambda_{0}^{-4}$, characteristic of Rayleigh-Brillouin scattering, and a factor of $\lambda_{0}^{-1} \propto \omega$ arising from the frequency spectrum of zero point fluctuations. This cross section was derived assuming that the initial and final photon states are plane waves.

In this Rapid Communication, our primary interest will be a related, but distinct effect which depends crucially upon the initial photon state being a localized wave packet. We will examine a space and time average of the squared density operator, $\hat{\rho}_{1}^{2}$, or equivalently of $\dot{\phi}^{2}$. This averaging will be determined by the details of the wave packet and the scattering 
measurement, and will give an additional contribution to the photon scattering. Consider the average of the squared density operator over finite regions of space and time in the form

$$
\overline{:{\hat{\rho_{1}}}^{2}(t, \mathbf{x}):}=\int d t f(t) \int d^{3} x g(\mathbf{x})\left[:{\hat{\rho_{1}}}^{2}(t, \mathbf{x}):\right] .
$$

Here we have normal ordered this operator, as we are here concerned with its fluctuations around its mean value. The functions $f(t)$ and $g(\mathbf{x})$ are sampling functions in time and space, respectively, and are assumed to have compact support, meaning that they vanish outside of finite intervals. These functions will model the measurement process of the density fluctuations, which is assumed to occur in a finite spacetime region. A class of such functions was constructed in Refs. [2,3], and may be characterized by their asymptotic forms of their Fourier transforms:

$$
\hat{f}(\omega) \sim e^{-|\omega \tau|^{\alpha}}, \quad \omega \tau \gg 1, \quad 0<\alpha<1
$$

and

$$
\hat{g}(\mathbf{k}) \sim e^{-(k \ell)^{\lambda}}, \quad k \ell \gg 1, \quad 0<\lambda<1,
$$

where we take the spatial function to be spherically symmetric for simplicity. Here $\tau$ and $\ell$ are the characteristic time and space scales for the sampling, so $f(t)$ is nonzero over a time interval of order $\tau$, and similarly $g(\mathbf{x})$ is nonzero in a sphere whose diameter is of order $\ell$. The constants $\alpha$ and $\lambda$ determine the rates of switch-on and switch-off, with smaller values corresponding to more rapid switching. The detailed relation between the value of $\alpha$ and the switch-on behavior of $f(t)$ was described in Sec. II D of Ref. [2]. A simple electrical circuit was also given in Ref. [2] in which the current increases after a switch is closed in accordance with the $\alpha=1 / 2$ function, so this is a physically realizable case.

Let $\delta \rho^{2}=\overline{\left(:{\hat{\rho_{1}}}^{2}:\right)} / \rho_{0}^{2}$ be the averaged fractional squared density fluctuations. The second moment, also the variance, of this operator is, in the continuum limit,

$$
\begin{aligned}
\mu_{2}= & \left\langle\left(\delta \rho^{2}\right)^{2}\right\rangle=\frac{\hbar^{2}}{128 \pi^{6} c_{s}^{4} \rho_{0}} \int d^{3} q_{1} d^{3} q_{2} \omega_{1} \omega_{2} \\
& \times \hat{f}^{2}\left(\omega_{1}+\omega_{2}\right) \hat{g}^{2}\left(\mathbf{q}_{1}+\mathbf{q}_{2}\right),
\end{aligned}
$$

which depends upon the details of the sampling functions. As discussed in Ref. [3], time averaging is essential for the fluctuations to be finite. If we were to let $f(t)=\delta(t)$, so that $\hat{f}(\omega)=1$, then $\mu_{2}$ would diverge due to a large contribution from modes with $\mathbf{q}_{1} \approx-\mathbf{q}_{\mathbf{2}}$.

Here we assume that the sampling functions arise from the shape of a probe wave packet of light, which propagates through the fluid, and measures the density fluctuations by light scattering. We take the packet to be approximately spherical with a size of about $\ell$, so it takes a time of about $\tau \approx \eta \ell / c$ to travel a distance $\ell$, as the speed of light in the fluid is $c / \eta$. If we are able to detect the photons scattered from the packet in this time, we have effectively measured $\delta \rho^{2}$. The root-mean-square value of a set of such measurements is predicted to be $\delta \rho_{\mathrm{rms}}^{2}=\sqrt{\mu_{2}}$. Because the speed of light is orders of magnitude larger than that of sound, we have $\ell=c \tau / \eta \gg c_{s} \tau$. In this case, we find

$$
\mu_{2} \approx \frac{(\hbar I)^{2}}{2^{8} \pi^{4} \rho_{0}^{2} \ell^{3} \tau^{5} c_{s}^{7}}
$$

where $I^{2}$ is the dimensionless integral

$$
I^{2}=\int_{0}^{\infty} d v v^{2} \hat{g}^{2}(v / l) \int_{0}^{\infty} d u u^{4} \hat{f}^{2}(u / \tau) .
$$

Now we can write

$$
\delta \rho_{\mathrm{rms}}^{2}=\sqrt{\mu_{2}} \approx \frac{\hbar I}{2^{4} \pi^{2} \rho_{0} \ell^{4}} \sqrt{\frac{c^{5}}{\eta^{5} c_{s}^{7}}},
$$

where in the final expression we have set $\tau=\eta \ell / c$. A crucial feature of this result is that $\delta \rho_{\mathrm{rms}}^{2} \propto \ell^{-4}$. This means that the fluctuations of the squared density increase rapidly when probed on smaller scales. Note that the variance $\mu_{2}$ also depends upon the shape of the wave packet through the dependence of $I^{2}$ on the parameters $\alpha$ and $\lambda$. In general, we can expect that smaller values of these parameters, corresponding to more rapid switching and a slower rate of decrease of $\hat{f}$ and $\hat{g}$, will lead to a larger variance.

The right-hand sides of both Eqs. (9) and (11) are leading terms in asymptotic expansions in powers of $c_{s} / c$, as well as being excellent approximations to the exact values for our problem. We can also calculate the third moment, $\mu_{3}$, to find that

$$
\frac{\left(\mu_{3}\right)^{1 / 3}}{\left(\mu_{2}\right)^{1 / 2}} \propto\left(\frac{c_{s} \tau}{\ell}\right)^{1 / 2} \ll 1 .
$$

The above ratio is small because $\ell \gg c_{s} \tau$, so the probability distribution for $\delta \rho^{2}$ is only very slightly skewed.

The factor of $1 / \tau^{5}$ in Eq. (9) is a reflection of the need for time averaging, which was discussed above. This factor in turn causes $\delta \rho_{\mathrm{rms}}^{2}$ in Eq. (11) to be proportional to the very large dimensionless ratio, $\left(c / c_{s}\right)^{5 / 2}$, which greatly enhances the magnitude of the squared density fluctuations in the fluid. Note that the density fluctuations we consider are large because of this factor which enhances the variance. Here we do not address the asymptotic form of the probability distribution, which depends upon higher moments.

Let us consider a wave packet of light propagating through the fluid, as illustrated in Fig. 1. We take the spatial size of the packet in all directions to be of order $\ell$, and its peak vacuum wavelength to be $\lambda_{0} \ll \ell$. Here $\eta$ is the index of refraction of the fluid at the peak wavelength, and is assumed to be of order one. We wish to consider photons scattered from this packet in a time of $\tau=\eta \ell / c$, during which the packet moves a distance of order $\ell$, so the scattering volume $\mathcal{V}$ will be of order $\ell^{3}$. The continuum approximation which we use seems to require that both $\lambda_{0}$ and $c_{s} \tau$ be larger than the interatomic spacing. In particular, we take

$$
c_{s} \tau \gtrsim 10^{10} \mathrm{~m} \text {. }
$$

Let $n_{\gamma}$ be the approximate number of photons in the packet, so the photon number flux is of order $n_{\gamma} /\left(\tau \ell^{2}\right)$. First consider the scattering effect described by Eq. (4). The mean number of scattered photons in time $\tau$ is about

$$
n_{s} \approx n_{\gamma} \frac{368(\pi n)^{4} \hbar \ell}{105 c_{s} \rho_{0} \lambda_{0}^{5}}
$$




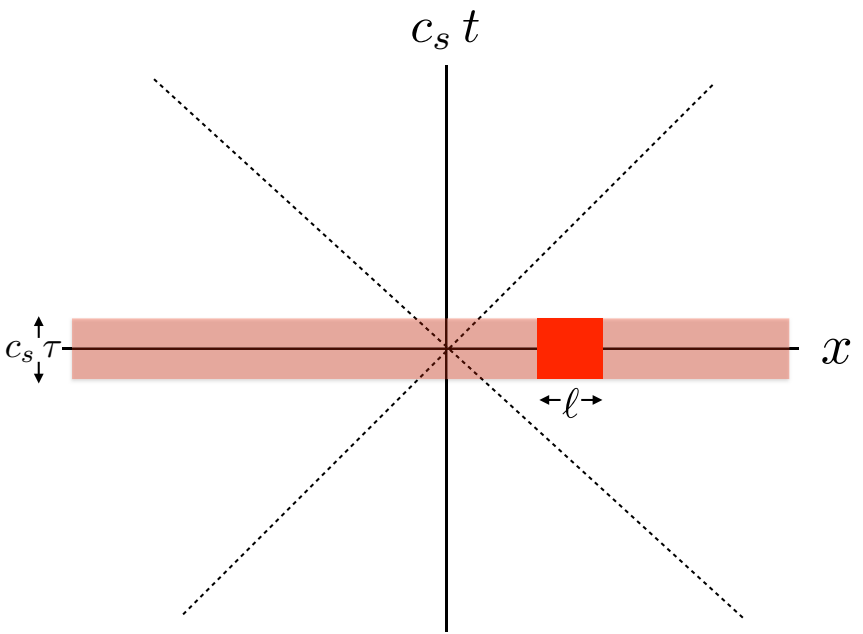

FIG. 1. A space-time diagram for the fluid. The dotted lines are the soundcone, the space-time paths of sound waves. The lightly shaded region is the space-time path of a light pulse, which moves in the $+x$ direction much more rapidly than sound. Here the pulse has a temporal duration of about $\tau$. The darker shaded region, whose spatial size is about $\ell$, represents the region which will scatter photons in a particular measurement.

Because Eq. (4) is a total cross section, $n_{s}$ includes photons scattered at all angles. The differential cross section from which Eq. (4) is derived [Eq. (23) in Ref. [8]] is proportional to $\sqrt{1-\cos \theta}$, where $\theta$ is the scattering angle. This means that backscattering, $\theta \approx \pi$, is somewhat more probable than scattering in other directions. Note that $n_{s}$ depends linearly upon $\ell$, but this is simply due to the increased scattering volume as $\ell$ increases. In contrast, $n_{s}$ increases as the the wavelength, $\lambda_{0}$, decreases. We interpret $n_{s}$ as a mean number of scattered photons, and $\delta \rho_{\text {rms }}^{2}$ as producing fluctuations around this mean value. Note that Eq. (4) and hence Eq. (14) strictly assume an initial plane-wave state with wavelength $\lambda_{0}$. However, if our wave packet has a spatial size $\ell \gg \lambda_{0}$, then the bandwidth will be small, $\Delta \lambda \ll \lambda_{0}$, and Eq. (4) is still applicable.

We may estimate the magnitude of the fluctuations due to $\delta \rho_{\text {rms }}^{2}$ by first considering an inhomogeneous medium with a local variation in density on a length scale $\ell$, which scatters light with a much shorter wavelength. Model the boundary between the region of varied density and the background medium as approximately a plane interface, and use the wellknown results for Fresnel scattering at such an interface. Let $\delta \eta$ be the fractional variation in index of refraction. Then the reflection probability for normal incidence is about $(\delta \eta)^{2} / 4$ for both polarizations, and for wave packets as well as plane waves if the dispersion is small. At other incidence angles, there is some dependence upon both the scattering angle and the polarization, but we will use the normal incidence results for an estimate. Note that if the magnitude of $\delta \eta$ is small, it is also approximately the fractional density variation. In our quantum treatment of density fluctuations, the classical fractional density variation is replaced by $\hat{\rho}_{1} / \rho_{0}$, so $(\delta \eta)^{2}$ becomes $\delta \rho_{\mathrm{rms}}^{2}$. In the case of a pulse of light containing $n_{\gamma}$ photons, the expected variation in the number of photons

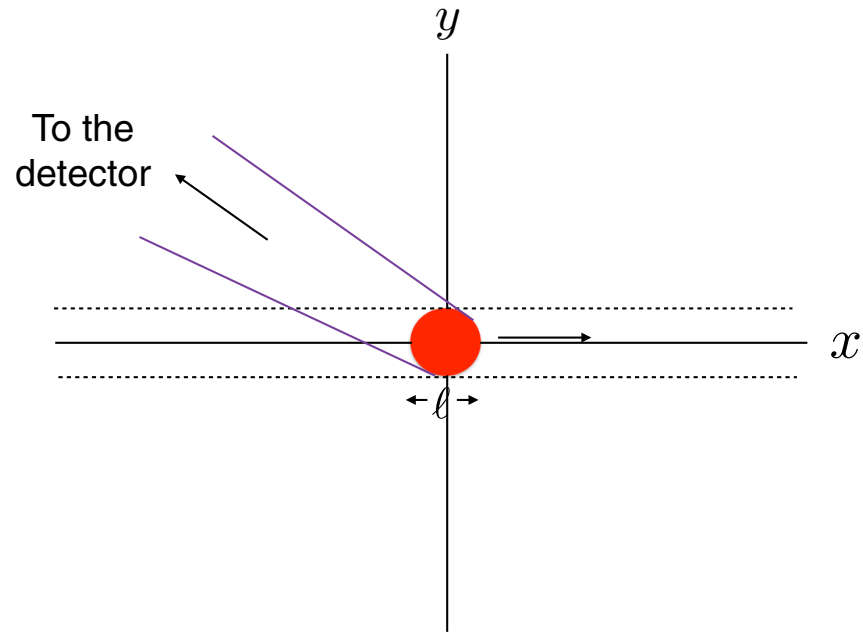

FIG. 2. The path of a wave packet of spatial size $\ell$ moving in the $+x$ direction is illustrated by the dotted lines. The shaded region is the approximate position of the wave packet during a time interval of about $\tau$, and is also the fluctuation region from which approximately backscattered photons will be detected.

scattered by a density fluctuation becomes

$$
\Delta n_{s} \approx \frac{1}{4} n_{\gamma} \delta \rho_{\mathrm{rms}}^{2} .
$$

The scattering of a wave packet by a local density fluctuation is illustrated in Fig. 2. In our view, the number of scattered photons, averaged over many trials, will be $n_{s}$, given by Eq. (14). However, in any one trial, the number of scattered photons is likely to differ from $n_{s}$ by about $\Delta n_{s}$. This distribution will be skewed very slightly toward numbers larger than $n_{s}$, but smaller numbers of scattered photons are almost equally probable in light of Eq. (12), although the associated probability distribution must vanish as the number of scattered photons goes to zero. We can view $\Delta n_{s}$ as arising from modifications of the phonon vacuum fluctuations due to the averaging produced by the finite wave packet size and shape, and the choice to select photons scattered in a finite region, as illustrated in Fig. 2.

We may combine Eqs. (11), (14), and (15) to obtain an expression for the expected fractional variation in scattered photon number due to the modified vacuum fluctuations:

$$
\frac{\Delta n_{s}}{n_{s}} \approx \frac{105 I c^{5 / 2} \lambda_{0}^{5}}{23 \times 2^{10} \pi^{6} c_{s}^{5 / 2} \eta^{13 / 2} \ell^{5}} .
$$

Before we discuss the possibility of observing this ratio, we need to consider statistical and thermal fluctuations. Let $\Delta n_{\text {stat }}$ be the expected statistical variation, so

$$
\frac{\Delta n_{\text {stat }}}{n_{s}}=\frac{1}{\sqrt{n_{s}}} \approx \sqrt{\frac{105 c_{s} \rho_{0} \lambda_{0}^{5}}{368(\pi \eta)^{4} n_{\gamma} \hbar \ell}},
$$

which leads to

$$
\frac{\Delta n_{s}}{\Delta n_{\text {stat }}} \approx \frac{I}{2^{8} \pi^{4} c_{s}^{3}} \sqrt{\frac{105 n_{\gamma} \hbar c^{5} \lambda_{0}^{5}}{23 \rho_{0} \ell^{9} \eta^{9}}} .
$$


The ratio between the differential cross sections of zero point and thermal fluctuations was found in Ref. [8] to be

$$
\frac{(d \sigma / d \Omega)_{\mathrm{ZP}}}{(d \sigma / d \Omega)_{\text {thermal }}} \approx \sqrt{2(1-\cos \theta)} \frac{\hbar \pi c_{s}}{\lambda_{0} k_{B} T} \eta^{4}\left(\eta^{2}-1\right)^{-2},
$$

where $T$ is temperature and $k_{B}$ is Boltzmann's constant. If we set $\theta=\pi$, for backscattering in the above expression, we can obtain an estimate of the ratio of $n_{s}$ to $n_{T}$, the expected number of photons scattered by thermal density fluctuations:

$$
\frac{n_{s}}{n_{T}} \approx \frac{2 \hbar \pi c_{s}}{\lambda_{0} k_{B} T} \eta^{4}\left(\eta^{2}-1\right)^{-2}
$$

which implies

$$
\frac{\Delta n_{s}}{n_{T}} \approx \frac{105 \hbar c^{5 / 2} \lambda_{0}^{4} I}{23 \times 2^{9} \pi^{5} c_{s}^{3 / 2} k_{B} T \eta^{5 / 2}\left(\eta^{2}-1\right)^{2} \ell^{5}} .
$$

The constant $I$ depends upon the choice of sampling functions. For a specific estimate, we set $\hat{f}(\omega)=\hat{h}_{\mathrm{fit}}(\omega)$ and $\hat{g}(k)=$ $\hat{g}_{\text {fit }}(k)$, where $\hat{h}_{\text {fit }}(\omega)$ and $\hat{g}_{\text {fit }}(k)$ are defined in Appendix A of Ref. [3], and correspond to $\alpha=\lambda=1 / 2$ in Eqs. (6) and (7). This choice gives $I \approx 113$.

Now we wish to give some numerical estimates for a specific liquid, which we take to be $\mathrm{He}^{3}$ in its normal (nonsuperfluid) phase. At $T=1 \mathrm{~K}$ and atmospheric pressure, $c_{s} \approx$ $200 \mathrm{~m} / \mathrm{s}[9], \rho_{0} \approx 83 \mathrm{~kg} / \mathrm{m}^{3}$ [10], and $\eta \approx 1.026[11,12]$. Furthermore, in the temperature interval $0.1 \mathrm{~K} \lesssim T \lesssim 1 \mathrm{~K}$, both $c_{s}$ and $\rho_{0}$ were found in Ref. [13] to be approximately independent of $T$. Given that the index of refraction is determined by $\rho_{0}$ and the atomic polarizability through the Claussius-Mossotti relation, we can expect $\eta$ also to be approximately independent of $T$. The lower bound on $\ell$ from the validity of the continuum limit, Eq. (13), is $\ell \gtrsim 150 \mu \mathrm{m}$, so let us consider a wave packet of length $\ell \approx 400 \mu \mathrm{m}$. For a wave packet of energy $E=1 \mu \mathrm{J}$ peaked at a wavelength of $1 \mu \mathrm{m}$, there are around $n_{\gamma}=5 \times 10^{12}$ photons. With this data, we find

$$
\begin{gathered}
\frac{\Delta n_{s}}{n_{s}} \approx 0.12\left(\frac{400 \mu \mathrm{m}}{\ell}\right)^{5}\left(\frac{1.026}{\eta}\right)^{13 / 2}\left(\frac{200 \mathrm{~m} / \mathrm{s}}{c_{s}}\right)^{5 / 2}\left(\frac{\lambda_{0}}{1 \mu \mathrm{m}}\right)^{5}, \\
\frac{\Delta n_{s}}{\Delta n_{\text {stat }}} \approx 8\left(\frac{400 \mu \mathrm{m}}{\ell}\right)^{9 / 2}\left(\frac{1.026}{\eta}\right)^{9 / 2}\left(\frac{200 \mathrm{~m} / \mathrm{s}}{c_{s}}\right)^{3}\left(\frac{\lambda_{0}}{1 \mu \mathrm{m}}\right)^{3}\left(\frac{E}{1 \mu \mathrm{J}}\right)^{1 / 2}\left(\frac{83 \mathrm{~kg} / \mathrm{m}^{3}}{\rho_{0}}\right)^{1 / 2},
\end{gathered}
$$

and

$$
\frac{\Delta n_{s}}{n_{T}} \approx 4.5\left(\frac{400 \mu \mathrm{m}}{\ell}\right)^{5}\left(\frac{200 \mathrm{~m} / \mathrm{s}}{c_{s}}\right)^{3 / 2}\left(\frac{\lambda_{0}}{1 \mu \mathrm{m}}\right)^{4}\left(\frac{0.1 \mathrm{~K}}{T}\right)
$$

with $\eta \approx 1.026$. In this case, we find that both the statistical and thermal fluctuation effects for $T \lesssim 0.5 \mathrm{~K}$ can be subdominant. Furthermore, $\Delta n_{s}$ can be a reasonable fraction of $n_{s}$, so it seems that the effects of space and time averaging might be observable.

In summary, we have argued that there are two contributions to light scattering by zero point density fluctuations in a liquid. One gives an average number of scattered photons and depends upon an inverse power of the photon wavelength. Apart from the wavelength dependence, this average is independent of the size and shape of the photon wave packets, and hence of the details of the measurement process. The other effect, the principal topic of this Rapid Communication, describes large fluctuations around this average, and depends upon an inverse power of the size of the scattering region, which is determined by the size and shape of the wave packets and our choice of which scattered photons to count in a given measurement. This effect is closely analogous to the quantum stress tensor fluctuations expected in relativistic quantum field theory, and is a vivid illustration of the deep role of the measurement process in quantum theory. The observation of both effects would reveal subtle, but distinct, features of quantum fluctuations.

We would like to thank Norman Birge, Chris Fewster, and Roger Tobin for helpful discussions. This work was supported in part by the National Science Foundation under Grant No. PHY-1912545.
[1] C. J. Fewster, L. H. Ford, and T. A. Roman, Probability distributions for quantum stress tensors in four dimensions, Phys. Rev. D 85, 125038 (2012).

[2] C. J. Fewster and L. H. Ford, Probability distributions for quantum stress tensors measured in a finite time interval, Phys. Rev. D 92, 105008 (2015).

[3] C. J. Fewster and L. H. Ford, Probability distributions for space and time averaged quantum stress tensors, Phys. Rev. D 101, 025006 (2020).

[4] See, for example, B. L. Hu and E. Verdaguer, Stochastic gravity: Theory and applications, Living Rev. Relativ. 7, 3 (2004).
[5] See, for example, E. M. Lifshitz and L. P. Pitaevski, Statistical Physics, Part 2, 2nd ed. (Pergamon, Oxford, 1969), Eq. (24.10).

[6] W. G. Unruh, Experimental Black-Hole Evaporation? Phys. Rev. Lett. 46, 1351 (1981); Sonic analog of black holes and the effects of high frequencies on black hole evaporation, Phys. Rev. D 51, 2827 (1995).

[7] P. O. Fedichev and U. R. Fischer, Cosmological quasiparticle production in harmonically trapped superfluid gases, Phys. Rev. A 69, 033602 (2004).

[8] L. H. Ford and N. F. Svaiter, Quantum Density Fluctuations in Classical Liquids, Phys. Rev. Lett. 102, 030602 (2009). 
[9] J. H. Vignos and H. A. Fairbank, Sound measurements in liquid and solid $\mathrm{He}^{3}, \mathrm{He}^{4}$, and $\mathrm{He}^{3}-\mathrm{He}^{4}$ mixtures, Phys. Rev. 147, 185 (1966).

[10] R. H. Sherman and F. J. Edeskuty, Pressure-VolumeTemperature relations of liquid $\mathrm{He}^{3}$ from 1.00 to $3.30{ }^{\circ} \mathrm{K}$, Ann. Phys. (NY) 9, 522 (1960).

[11] M. H. Edwards, Refractive index of $\mathrm{He}^{4}$ : Liquid, Can. J. Phys. 36, 884 (1958).
[12] C. E. Chase, E. Maxwell, and W. E. Millett, The dielectric constant of liquid helium, Physica 27, 1129 (1961).

[13] B. M. Abraham and D. W. Osborne, Experimental determination of the molar volume and derivation of the expansion coefficient, entropy change on compression, compressibility, and first-sound velocity for liquid ${ }^{3} \mathrm{He}$ from 35 to $1200 \mathrm{mK}$ and from the saturation pressure to 24 atm, J. Low Temp. Phys. 5, 335 (1971). 